\title{
Idiopathic Intracranial Hypertension in Pregnancy Treated with Serial Lumbar Punctures
}

\author{
Manasi Badve ${ }^{1}$, Matthew J. McConnell ${ }^{3}$, Tanmay Shah ${ }^{1}$, Kristin M. Ondecko-Ligda ${ }^{1}$, George W. \\ Poutous $^{2}$, Manuel C. Vallejo ${ }^{1}$
}

${ }^{1}$ Department of Anesthesiology, Magee-Womens Hospital of UPMC, Pittsburgh, USA; ${ }^{2}$ Department of Obstetrics, Gynecology and Reproductive Medicine, Magee-Womens Hospital of UPMC, University of Pittsburgh, Pittsburgh, USA; ${ }^{3}$ Department of Anesthesiology, Ohio State University Medical Center, Columbus, USA.

Email: vallejomc@anes.upmc.edu

Received September $23^{\text {rd }}, 2010$; revised December $14^{\text {th }}, 2010$; accepted December $21^{\text {st }}, 2010$.

\begin{abstract}
Idiopathic intracranial hypertension $(\mathrm{IIH})$ is a syndrome of elevated intracranial pressure with normal CSF composition and no evidence of hydrocephalus or mass lesion. We describe the anesthetic management of a parturient with IIH who required multiple lumbar punctures during pregnancy and delivery secondary to worsening neurological symptoms.
\end{abstract}

Keywords: Pregnancy, Idiopathic Intracranial Hypertension, Lumbar Puncture, Cesarean Section

\section{Introduction}

Idiopathic intracranial hypertension (IIH) formerly known as pseudotumor cerebri or benign intracranial hypertension is a syndrome of elevated intracranial pressure with normal CSF composition and no evidence of hydrocephalus or mass lesion. It is typically seen in obese women of childbearing age with an incidence of 19.3 per 100,000 . Cases presenting during pregnancy have symptoms similar to non-pregnant women and pregnancy in turn does not increase the risk of IIH [1]. Most common presentations include headache, transient visual obscurations and pulsatile tinnitus. Other symptoms such as neck pain, photopsia, shoulder or back discomfort, nausea and vomiting reflecting meningeal irritation may be present. Associated signs include papilledema on funduscopic examination and ophthalmoplegia due to sixth cranial nerve involvement. Diagnosis is by exclusion using modified Dandy criteria (Table 1) which require an increased intracranial pressure greater than $250 \mathrm{~mm}$ of water and ruling out other pathologies by CSF examination and radiological imaging [2].

We describe the anesthetic management of a young woman with IIH during her second pregnancy that required multiple lumbar punctures throughout the pregnancy secondary to worsening neurological symptoms.

\section{Case Description}

A 20 year-old G2P1 African-American female at 17 weeks gestation, with a pre-pregnancy BMI of $39 \mathrm{~kg} / \mathrm{m}^{2}$ (height $152.5 \mathrm{~cm}$ and weight $91 \mathrm{~kg}$ ) presented to the emergency room with complaints of increasing dizziness, headache and lightheadedness of 10 days duration. She had premature rupture of the membranes during her first pregnancy followed by an uncomplicated vaginal delivery. Past medical history was significant for hydronephrosis and multiple urinary tract infections treated with nitrofurantoin in this pregnancy. Past surgical history included an uneventful laparoscopic cholecystectomy. She was diagnosed with mild dehydration, given fluids and discharged to home.

At $24 \frac{1}{2}$ weeks gestation, the patient was seen by her obstetrician with continued complaints of headache, visual disturbances and neck pain. She was referred to a neurologist who found significant blurring of the optic nerve margins. The neurologist established a diagnosis of $\mathrm{IIH}$ and immediately directed her to the emergency room to have a lumbar puncture performed, which was unsuc-

Table1. Modified Dandy Criteria [2].

\footnotetext{
1. Signs and/or symptoms of increased intracranial pressure.

2. Documented elevated intracranial pressure $(>250 \mathrm{~mm}$ of water).

3. Normal CSF composition.

4. No evidence of hydrocephalus, mass, structural, or vascular lesion on imaging.

5. No localizing neurological signs except a unilateral/ bilateral VI nerve paresis.
}

(Adapted from Friedman and Jacobson) 
cessful. She was then referred to Magee-Womens Hospital $(\mathrm{MWH})$ for the procedure. As a tertiary care referral hospital, MWH has approximately 10,000 deliveries per year with a $90 \%$ neuraxial block placement rate and expertise in performing spinal anesthetics. In addition, the use of neuraxial ultrasound for visualization would be available for lumbar puncture if necessary. The neurologist recommended the CSF pressure be decreased to approximately $200 \mathrm{~mm}$ of $\mathrm{H}_{2} \mathrm{O}$. At $\mathrm{MWH}$, a lumbar puncture was performed successfully with a $20 \mathrm{~g}$ Whitacre needle in L4-5 vertebral interspace using an aseptic technique on the first attempt. The opening CSF pressure was measured at $380 \mathrm{~mm} \mathrm{H} \mathrm{H}_{2} \mathrm{O}$ and was reduced to $220 \mathrm{~mm}$ $\mathrm{H}_{2} \mathrm{O}$ after drainage of $14 \mathrm{~mL}$ of CSF. Total time for CSF drainage was approximately 45 minutes. She reported immediate relief of her visual symptoms after the procedure. However, the patient experienced a post-dural puncture headache (PDPH) requiring overnight admission. She was discharged the next morning with resolution of her PDPH and no further incident. It was decided by anesthesiology to use a smaller needle in the future in order to prevent PDPH. She was seen by her neurologist again at 26 weeks, ten days after having her first lumbar puncture. At that time she reported minor back pain from the lumbar puncture, but generally was feeling much better. She denied visual symptoms and headache. Optic nerve borders were noted to be more distinct bilaterally and her papilledema appeared to be improving. The recommendation was made for the patient to have a scheduled cesarean section to avoid bearing down during vaginal delivery.

At 31 weeks gestation, the patient was seen again by her neurologist for returning symptoms. She also reported mild but worsening dizziness and pressure at the base of her neck that abated with sitting. A repeat LP was scheduled with her anesthesiologist at MWH at 36 weeks gestation. The patient continued to have neck pain, blurred vision, intermittent blindness, lightheadedness, generalized weakness, nausea and vomiting at that appointment. The lumbar puncture was performed successfully with a $25 \mathrm{~g}$ needle and an opening pressure of 400 $\mathrm{mm} \mathrm{H}_{2} \mathrm{O}$ reduced to $180 \mathrm{~mm} \mathrm{H}_{2} \mathrm{O}$ after drainage of 22 $\mathrm{mL}$ of CSF. She subsequently noted relief of symptoms and reported no post-dural puncture headache.

At 38 weeks gestation, the fetal lungs were determined to be mature via amniocentesis, with a surfactantalbumin ratio of $83 \mathrm{mg} / \mathrm{g}$. She was scheduled for an elective cesarean section to be performed two days later. On the day of her surgery, the patient once again complained of dizziness and blurred vision. The patient was taken to the operating room and standard monitors including a cardioscope, non-invasive blood pressure cuff and pulseoximeter were applied. A lumbar puncture was placed as before. The opening CSF pressure was $440 \mathrm{~mm}$ $\mathrm{H}_{2} \mathrm{O}$ and reduced to $200 \mathrm{~mm} \mathrm{H}_{2} \mathrm{O}$ with the removal of $20 \mathrm{~mL}$ of CSF. After CSF removal, $12 \mathrm{mg}$ of hyperbaric bupivacaine with $0.2 \mathrm{mg}$ preservative free morphine and $20 \mathrm{mcg}$ fentanyl was injected into the intrathecal space using the needle already in place. The cesarean section proceeded uneventfully with delivery of a healthy boy without complications. Total blood loss was $700 \mathrm{ml}$ and the patient received $1000 \mathrm{ml}$ of lactated Ringer's fluid. Total urine output during the procedure was $150 \mathrm{~mL}$. APGAR scores were 9 and 9 at 1 and 5 minutes postdelivery, respectively.

The patient was discharged home on the third postoperative day after an uneventful stay in the hospital. She was seen by her neurologist one week later and had no complaints of headache, neck pain, or visual disturbances. She has had no recurrence of signs or symptoms of IIH since her delivery. The patient opted to have an Essure tubal ligation, which was performed successfully three months after delivery.

\section{Discussion}

With a prevalence rate of $2-12 \%$, pregnancy in women with IIH gives rise to both diagnostic and therapeutic limitations [1]. This is due to the concern of the deleterious effects of the radiological imaging and teratogenicity of drugs in the growing fetus.

Ophthalmoscopic examination for papilledema, perimetry to detect visual field loss and a diagnostic lumbar puncture to measure CSF pressures can be performed in a pregnant patient suspected of having IIH. But imaging modalities like computed tomography and contrast enhanced MRI should be performed after thorough patient counseling regarding the risks and benefits [3].

It is postulated that thrombophilia and hypofibrinolysis seen in high estrogen conditions (pregnancy, obesity, polycystic ovarian syndrome, exogenous estrogen administration lead to thrombosis of arachnoid villi and reduced CSF absorption [4]. Bagga et al report 3 likely cases of IIH in pregnancy. All the women in their series were obese and presented with features suggestive of IIH during pregnancy, which resolved postpartum. According to them, symptoms of IIH may appear or worsen during pregnancy and usually disappear after delivery which was seen in our patient [5].

Our patient initially presented at 17 weeks gestation with headache, visual disturbances, and neck pain as her primary symptoms. Her visual disturbance was the most worrisome symptom, as it indicated involvement of the optic nerve and could worsen to cause a permanent deficit $[6,7]$. The ultimate goals of her treatment were to alleviate symptoms and prevent permanent visual loss. Although, a variety of medical and surgical options are 
available to treat IIH, they must be considered based on the individual clinical scenario in which it is to be used.

Medical management includes weight control, drugs to reduce intracranial pressure and repeated lumbar punctures. In non-pregnant patients, diet and weight loss are considered integral parts of IIH treatment, with even modest loss showing improvement of symptoms [8]. In pregnancy, however, the goal should be weight control while avoiding ketosis, with gain limited to 20 pounds [3]. The role of dietary control in pregnancy is to limit weight gain so as not to worsen the symptoms while allowing for adequate fetal growth. Because of the immediacy of required treatment, dietary control was encouraged but not sufficient in the case presented here.

Acetazolamide, a carbonic anhydrase inhibitor is widely considered the first line medical treatment for IIH, alleviating symptoms by decreasing the CSF production and hence intracranial pressure [9]. Carbonic anhydrase inhibitors have been found to produce limb anomalies in rodents and there are no convincing reports of well controlled studies in pregnant women. It is classified as the category $\mathrm{C}$ drug in pregnancy and its safety in human pregnancy has not been confirmed $[3,10]$. Due to the rapidity of her worsening symptoms and potential risks of acetazolamide compounded by the parturient's chronic renal dysfunction, serial lumbar punctures were preferentially chosen for treatment to relieve symptoms without increased risk to the fetus.

Other medical treatment options besides acetazolamide that may be indicated for IIH include furosemide, topiramate, and corticosteroids $[6,9,11]$. Furosemide reduces intracranial pressure by both diuresis and reducing the transport of sodium ions in the brain. Like acetazolamide, it has been classified by FDA as the category $\mathrm{C}$ drug $[3,10]$. The use of furosemide for IIH during pregnancy is recommended only when potential benefits justify the potential risks and that too for a short period of time [3].

Topiramate may also be efficacious and well tolerated in the treatment of IIH because of its carbonic anhydrase activity and weight reducing effect $[8,9,11,12]$. However, side effects include nephrolithiasis and fetal deformities [13]. These factors limited its use in our patient.

Corticosteroids are classified as a category B risk factor for pregnancy and recommended only for short term use in acute visual decline [3,7]. Steroids have been found to cause lip and palate defects in animals. They may provide symptomatic improvement by presumed reduction of brain swelling [14]. We avoided using corticosteroids to prevent early fetal lung maturation.

Lumbar puncture (LP) is an effective management tool in pregnancy with IIH as it directly and immediately reduces intracranial pressure. In patients with an increased intracranial pressure because of a space occupying lesion, a lumbar puncture can cause herniation of brainstem. But in IIH, uniform swelling of brain prevents herniation and compression of medulla oblongata. It allows CSF drainage and reduces CSF pressure [14]. Repeated spinal fluid drainage by LP can be helpful for improvement of symptoms of IIH in pregnancy and preventing permanent vision loss [1,3,7], as was the case with our patient. Lumbar puncture is considered by some to be the 'treatment of choice' during pregnancy, and most patients experience improvement of symptoms for several days after a lumbar puncture. In this case, 11/2 weeks passed between the first and second lumbar puncture, a significantly longer period than that typically experienced in other reports $[3,14]$. Ultimately, serial lumbar punctures was an effective treatment choice in this parturient. Her response to the LPs was significant, rapid, and sustained. Success of treatment was also seen on ophthalmoscopic exam, as the papilledema improved and the borders of the optic nerve were noted to become more distinct after the procedure. The initial opening pressures in this case were 380,400 , and $440 \mathrm{~mm} \mathrm{H}_{2} \mathrm{O}$ on successive lumbar punctures; pressures after drainage of fluid were consistently below the $250 \mathrm{~mm} \mathrm{H}_{2} \mathrm{O}$ threshold, further validating the choice of treatment. The only adverse event of note regarding the serial LPs was one episode of a PDPH that was short-lived and was avoided on subsequent LPs with the use of a smaller needle.

When the medical line of treatment fails or if the vision deteriorates rapidly, surgical options may be considered. One of the mechanisms proposed for the association of obesity and IIH is that obesity increases the intraabdominal pressure which in turn raises the central venous pressures. This obstructs the cerebral venous return. Surgical procedures like optic nerve sheath fenestration and lumboperitoneal or ventriculoperitoneal shunting may improve the venous drainage from the brain $[6,8,15]$. Since IIH is associated with venous sinus hypertension, venous sinus stenting has also been proposed for unresponsive cases [16]. Of these procedures, optic nerve sheath fenestration requires less anesthesia time which is one of the factors that has to be considered in pregnant women [3].

Choice of anesthesia for a patient with IIH undergoing abdominal delivery also depends on whether she has had a CSF shunting procedure. Since our patient was primarily treated with repeated lumbar punctures, spinal anesthesia was considered to be the best option [14,17]. The block height achieved in this case was adequate for a cesarean section. General anesthesia with its associated risks of aspiration and airway problems, especially in an obese parturient was best avoided. However, general anesthesia may be required in a patient with a lumboperitoneal shunt in place, as there is a possibility that the 
spinal anesthetic may escape into the peritoneal cavity through the shunt leading to inadequate anesthesia and damage to the shunt during block placement [3]. Also, with both spinal and epidural block, radiological imaging may be needed to locate the exact position of the shunt to avoid damage [14].

With symptom control, our patient had a successful cesarean section. Overall, patients presenting with IIH in pregnancy have an excellent prognosis, though a minority may exhibit continued elevated ICP and associated symptoms after delivery [7]. Visual outcomes are similar to those for non-pregnant patients, with improvement and minimal long term losses with timely and appropriate treatment $[1,3]$. Our patient had no symptoms at the time of her delivery because of her LP being placed prior to her cesarean section, and continued to be symptom free at her follow-up appointments with her neurologist.

\section{REFERENCES}

[1] R. Huna-Baron and M. J. Kupersmith, "Idiopathic Intracranial Hypertension in Pregnancy," Journal of Neurology, Vol. 249, No. 8, 2002, pp. 1078-1081. doi:10.1007/s00415-002-0791-4

[2] D. I. Friedman and D. M. Jacobson, "Diagnostic Criteria for Idiopathic Intracranial Hypertension," Neurology, Vol. 59, No. 10, 2002, pp. 1492-1495.

[3] R. A. Tang, E. U. Dorotheo, J. S. Schiffman and H. M. Bahrani, "Medical and Surgical Management of Idiopathic Intracranial Hypertension in Pregnancy," Current Neurology and Neuroscience Reports, Vol. 4, No. 5, 2004, pp. 398-409. doi:10.1007/s11910-004-0087-4

[4] C. J. Glueck, D. Aregawi, N. Goldenberg, K. C. Golnik, L. Sieve and P. Wang, "Idiopathic Intracranial Hypertension, Polycystic-Ovary Syndrome, and Thrombophilia," Journal of Laboratory Clinical Medicine., Vol. 145, No. 2, 2005, pp. 72-82. doi:10.1016/j.lab.2004.09.011

[5] R. Bagga, V. Jain, K. R. Gupta, S. Gopalan, S. Malhotra and C. P. Das, "Choice of Therapy and Mode of Delivery in Idiopathic Intracranial Hypertension During Pregnancy," Medscape General Medicine, Vol. 7, No. 4, 2005, p. 42 .

[6] M. Wall, "Idiopathic Intracranial Hypertension," Neurologic Clinics, Vol. 9, No. 1, 1991, pp. 73-95.

[7] R. W. Evans and D. I. Friedman, "The Management of Pseudotumor Cerebri during Pregnancy," Headache, Vol.
40, No. 6, 2000, pp. 495-497. doi:10.1046/j.1526-4610.2000.00076.x

[8] S. Randhawa and G. P. Van Stavern, "Idiopathic Intracranial Hypertension (Pseudotumor Cerebri)," Current Opinion in Ophthalmology, Vol. 19, No. 6, 2008, pp. 445-453. doi:10.1097/ICU.0b013e3283112d3e

[9] N. Çelebisoy, F. Gökçay, H. Şirin and Ö. Akyürekli, "Treatment of Idiopathic Intracranial Hypertension: Topiramate vs Acetazolamide, an Open-Label Study," Acta Neurologica Scandinavica, Vol. 116, No. 5, 2007, pp. 322-327. doi:10.1111/j.1600-0404.2007.00905.x

[10] A. G. Lee, M. Pless, J. Falardeau, T. Capozzoli, M. Wall and R. H. Kardon, "The Use of Acetazolamide in Idiopathic Intracranial Hypertension during Pregnancy," American Journal of Ophthalmology, Vol. 139, No. 5, 2005, pp. 855-859. doi:10.1016/j.ajo.2004.12.091

[11] M. Wall, "Idiopathic Intracranial Hypertension (Pseudotumor Cerebri)," Current Neurology and Neuroscience Reports, Vol. 8, 2008, pp. 87-93. doi:10.1007/s11910-008-0015-0

[12] E. Palacio, L. Rodero and J. Pascual, "Topiramate-Re sponsive Headache Due to Idiopathic Intracranial Hypertension in Behcet Syndrome," Headache, Vol. 44, No. 5, 2004, pp. 436-437. doi:10.1111/j.1526-4610.2004.04095.x

[13] S. Hunt, A. Russell, W. H. Smithson, L. Parsons, I. Robertson, R. Waddell, B. Irwin, P. J. Morrison, J. Morrow and J. Craig, "Topiramate in pregnancy: Preliminary experience from the UK Epilepsy and Pregnancy Register," Neurology, Vol. 71, No. 4, 2008, pp. 272-276. doi:10.1212/01.wnl.0000318293.28278.33

[14] E. Abouleish, V. Ali and R. A. Tang, "Benign Intracranial Hypertension and Anesthesia for Cesarean Section," Anesthesiology, Vol. 63, No. 6, 1985, pp. 705-707. doi:10.1097/00000542-198512000-00029

[15] P. Brazis and A. Lee, "Elevated Intracranial Pressure and Pseudotumor Cerebri," Current Opinion in Ophthalmology, Vol. 9, No. 6, 1998, pp. 27-32. doi:10.1097/00055735-199812000-00005

[16] P. Brazis, "Pseudotumor Cerebri," Current Neurology and Neuroscience Reports, Vol. 4, No. 2, 2004, pp. 111-116. doi:10.1007/s11910-004-0024-6

[17] C. M. Peterson and J. V. Kelly, "Pseudotumor Cerebri in Pregnancy. Case Reports and Review of Literature," Obstetrical and Gynecological Survey, Vol. 40, No. 6, 1985, pp. 323-329. doi:10.1097/00006254-198506000-00001 